\title{
Maxwell's Equations as the Basis for Model of Atoms
}

\author{
Milan Perkovac \\ The First Technical School TESLA, Klaiceva 7, Zagreb, Croatia \\ Email: milan@drivesc.com
}

Received February 2014

\begin{abstract}
A century ago the classical physics couldn't explain many atomic physical phenomena. Now the situation has changed. It's because within the framework of classical physics with the help of Maxwell's equations we can derive Schrödinger's equation, which is the foundation of quantum physics. The equations for energy, momentum, frequency and wavelength of the electromagnetic wave in the atom are derived using the model of atom by analogy with the transmission line. The action constant $A_{0}=\left(\mu_{0} / \varepsilon_{0}\right)^{1 / 2} s_{0}{ }^{2} e^{2}$ is a key term in the above mentioned equations. Besides the other well-known constants, the only unknown constant in the last expression is a structural constant of the atom $s_{0}$. We have found that the value of this constant is 8.27756 and that it shows up as a link between macroscopic and atomic world. After calculating this constant we get the theory of atoms based on Maxwell's and Lorentz equations only. This theory does not require knowledge of Planck's constant $h$, which is replaced with theoretically derived action constant $A_{0}$, while the replacement for the fine structure constant $\alpha^{-1}$ is theoretically derived expression $2 s_{0}{ }^{2}=137.036$. So, the structural constant $s_{0}$ replaces both constants $h$ and $\alpha$. This paper also defines the stationary states of atoms and shows that the maximal atomic number is equal to $Z_{\max }=137$. The presented model of the atoms covers three of the four fundamental interactions, namely the electromagnetic, weak and strong interactions.
\end{abstract}

\section{Keywords}

Action Constant, Fine Structure Constant, Lecher's Line, Maxwell's Equations, New Elements, Phase Velocity, Planck's Constant, Stability of Atoms, Standing Waves, Stationary States, Synchronized States, System of the Elements, Structural Coefficient, Structural Constant, Transmission Line, Undiscovered Elements

\section{Introduction}

During the whole of twentieth century physics has mostly been searching for answers to fundamental questions of matter, primarily the relationship between waves and particles. Discussions on this have not yet been completed. It is believed that natural laws are exactly the same in the macro and micro world.

But this general approach to the macroscopic and microscopic scale seemed questionable in the case of Maxwell's equations. In fact, Maxwell's equations have reached excellent results in the macroscopic scale. Their ap- 
plication in the microscopic scale at first glances seemed disappointing. Actually, Maxwell's equations previously couldn't explain stability of atoms (with respect to the radiation energy of those particles which are moving with acceleration), the periodic table of elements, the chemical bond, the discrete excitation energies of atoms and their energetic state, the ionization of atoms, the spectra, including its fine structure and transition rules, experimental evidence about X-ray spectra and the behavior of atoms in electric and magnetic fields, as well as the properties of matter in solid state. That was the reason why classical physics failed when applied to the atomic area, i.e., to the area of nanometers or below. Thus entire physics divided into two branches, namely, traditional, classical physics and new, quantum physics.

Despite the uncertain physical meaning of the wave function $\psi$ at the famous Schrödinger's equation, $\nabla^{2} \psi+$ $8 \pi^{2} m(W-U) \psi / h^{2}=0$, the equation has played an important role in the development of quantum physics. The wave function $\psi(x, y, z)=\psi(\boldsymbol{r})$ is the solution of the Schrödinger equation, i.e., it is a mathematical expression involving coordinates $(x, y, z)$ of a particle in space, or expressed as a vector $\boldsymbol{r}=x \boldsymbol{i}+y \boldsymbol{j}+z \boldsymbol{k}$, where $\boldsymbol{i}, \boldsymbol{j}$ and $\boldsymbol{k}$ are unit vectors in the Cartesian coordinates (all vectors below are bold cursive letters). If we solved the Schrödinger equation for a particle in a given system then, depending on the boundary condition, the solution is a set of allowed wave functions of the particle, each corresponding to an allowed energy level. The usual interpretation of the wave function $\psi$ is that the square of its absolute value, i.e. $|\psi|^{2}$, at a given point is proportional to the probability of finding the particle in a small element of volume, $d x d y d z$, at that point. All of this was obtained without the use of Maxwell's equations, in the early twentieth century.

However, after the explanation of radiation [1-4], Maxwell's equations can now contribute to modern physics much more than before. From Maxwell's equations we derive Schrödinger's equation, which is the basis of quantum physics. New findings may lead to a new interpretation of Schrödinger’s equation.

The meaning of physical quantities in Maxwell's equations is completely clear. Therefore, the meanings of the wave functions of these equations, which are derived from Maxwell's equations, are also completely clear.

This paper describes many atomic phenomena with the help of classical physics and shows how Schrödinger's equation is obtained by using Maxwell's equations. In this way, the above-mentioned disadvantages of classical physics can now be explained in the framework of classical physics.

\section{Derivation of Wave Equations}

\subsection{Wave Equation in the Atom}

Maxwell's equations are the four differential equations describing the space and time, i.e., $(\boldsymbol{r}, t)$, dependence of the electromagnetic field [5-10]:

Gauss's law for electric flux (electric flux begins and ends on charge or at infinity);

$$
\nabla \cdot \boldsymbol{D}(\boldsymbol{r}, t)=\rho(\boldsymbol{r}, t)
$$

Gauss's law for magnetism (where magnetic field lines have no beginning or end);

$$
\nabla \cdot \boldsymbol{B}(\boldsymbol{r}, t)=0
$$

Faraday's law of electromagnetic induction; a changing $\boldsymbol{B}$ produce $\boldsymbol{E}$;

$$
\nabla \times \boldsymbol{E}(\boldsymbol{r}, t)=-\frac{\partial \boldsymbol{B}(\boldsymbol{r}, t)}{\partial t},
$$

Ampère's circuital law (with Maxwell's correction); $\boldsymbol{H}$ is produced by current $\boldsymbol{J}$ and by changing $\boldsymbol{D}$;

$$
\boldsymbol{\nabla} \times \boldsymbol{H}(\boldsymbol{r}, t)=\boldsymbol{J}(\boldsymbol{r}, t)+\frac{\partial \boldsymbol{D}(\boldsymbol{r}, t)}{\partial t}
$$

where $\boldsymbol{D}$ is electric displacement, $\boldsymbol{E}$ is electric field strength, $\boldsymbol{B}$ is magnetic flux density, $\boldsymbol{H}$ is the magnetic field strength, $\rho$ is volume charge density, and $\boldsymbol{J}$ is electric current density. Differential Hamilton's operator $\nabla=\boldsymbol{i} \partial / \partial x+\boldsymbol{j} \partial / \partial y+\boldsymbol{k} \partial / \partial z$ is called "del" or "nabla" operator is a symbolically vector.

There are a total of 16 variables in (1a)-(1d) (the 15 components of five vectors $\boldsymbol{E}, \boldsymbol{D}, \boldsymbol{B}, \boldsymbol{H}, \boldsymbol{J}$, and the scalar $\rho$ ). If the source densities $\rho$ and $\boldsymbol{J}$ are given (four known variables) there still remain 12 unknown variables. There are, however, eight equations; one from (1a), one from (1b), and three for components in (1c) and (1d) each. Obviously, to make this system determinate we need additional relations. 
For a completely linear medium there are constitutive relations describing the properties of the media in which the fields exist: $\boldsymbol{D}=\varepsilon \boldsymbol{E}, \boldsymbol{B}=\mu \boldsymbol{H}, \boldsymbol{J}=\boldsymbol{g} \boldsymbol{E}$, where $\varepsilon$ is permittivity, $\varepsilon=\varepsilon_{\mathrm{r}} \varepsilon_{0}$, where $\varepsilon_{\mathrm{r}}$ is relative permittivity, $\varepsilon_{0}$ is permittivity of free space, $\mu$ is permeability, $\mu=\mu_{\mathrm{r}} \mu_{0}$, where $\mu_{\mathrm{r}}$ is relative permeability, $\mu_{0}$ is permeability of free space, and $g$ is the conductivity of the media. Hence [without writing $(\boldsymbol{r}, t)$ which is implied] Maxwell equations become:

Gauss's law for electric flux;

$$
\nabla \cdot \boldsymbol{E}=\frac{\rho}{\varepsilon}
$$

Gauss's law for magnetism;

$$
\nabla \cdot \boldsymbol{B}=0
$$

Faraday’s law;

$$
\nabla \times \boldsymbol{E}=-\frac{\partial \boldsymbol{B}}{\partial t},
$$

Ampère's law;

$$
\nabla \times \boldsymbol{B}=\mu \boldsymbol{J}+\varepsilon \mu \frac{\partial \boldsymbol{E}}{\partial t} .
$$

1) Let's take curl on Faraday's law, i.e., on Equation (2c):

$$
\boldsymbol{\nabla} \times(\boldsymbol{\nabla} \times \boldsymbol{E})=-\frac{\partial(\boldsymbol{\nabla} \times \boldsymbol{B})}{\partial t} .
$$

On the other hand, for each vector $\boldsymbol{E}$ the following is true:

$$
\nabla \times(\nabla \times E)=\nabla(\nabla \cdot E)-\nabla^{2} \boldsymbol{E},
$$

and the substitution in Equation (3) Ampere's law from Equation (2d) gives:

$$
\nabla(\nabla \cdot \boldsymbol{E})-\nabla^{2} \boldsymbol{E}=-\frac{\partial}{\partial t}\left(\mu \boldsymbol{J}+\varepsilon \mu \frac{\partial \boldsymbol{E}}{\partial t}\right)
$$

where del-squared $\nabla^{2}=\frac{\partial^{2}}{\partial x^{2}}+\frac{\partial^{2}}{\partial y^{2}}+\frac{\partial^{2}}{\partial z^{2}}$ is an operator called the Laplacian. Now in Equation (5) add Gauss's law for electric flux, i.e., Equation (2a), and $\boldsymbol{J}=g \boldsymbol{E}$ :

$$
\nabla^{2} \boldsymbol{E}-\mu g \frac{\partial \boldsymbol{E}}{\partial t}-\varepsilon \mu \frac{\partial^{2} \boldsymbol{E}}{\partial t^{2}}-\nabla\left(\frac{\rho}{\varepsilon}\right)=0 .
$$

2) Let's take curl on Ampère's law, i.e., on Equation (2d):

$$
\nabla \times(\nabla \times \boldsymbol{B})=\nabla \times\left(\mu \boldsymbol{J}+\varepsilon \mu \frac{\partial \boldsymbol{E}}{\partial t}\right) .
$$

On the other hand, for each vector $\boldsymbol{B}$ the following is true:

$$
\nabla \times(\nabla \times B)=\nabla(\nabla \cdot B)-\nabla^{2} \boldsymbol{B} .
$$

Using $\boldsymbol{J}=g \boldsymbol{E}$ here gives:

$$
\nabla(\nabla \cdot \boldsymbol{B})-\nabla^{2} \boldsymbol{B}=g \mu(\nabla \times \boldsymbol{E})+\varepsilon \mu \frac{\partial(\boldsymbol{\nabla} \times \boldsymbol{E})}{\partial t} .
$$

Including Faraday's law from Equation (2c) into Equation (9) we get:

$$
\nabla(\nabla \cdot \boldsymbol{B})-\nabla^{2} \boldsymbol{B}=-g \mu \frac{\partial \boldsymbol{B}}{\partial t}-\varepsilon \mu \frac{\partial^{2} \boldsymbol{B}}{\partial t^{2}} .
$$

Using Equations (2b), i.e., $\boldsymbol{\nabla} \cdot \boldsymbol{B}=0$, and $\boldsymbol{B}=\mu \boldsymbol{H}$, and after sharing with $\mu$, we get from Equation (10) we 
get:

$$
\nabla^{2} \boldsymbol{H}-g \mu \frac{\partial \boldsymbol{H}}{\partial t}-\varepsilon \mu \frac{\partial^{2} \boldsymbol{H}}{\partial t^{2}}=0 .
$$

Equations (6) and (11) are the required wave equations. These two vector equations constitute six component equations with seven unknowns $\left(E_{\mathrm{x}}, E_{\mathrm{y}}, E_{\mathrm{z}}, H_{\mathrm{x}}, H_{\mathrm{y}}, H_{\mathrm{z}}, \rho\right)$. The system becomes determined if viewed without charge, i.e., when $\rho=0$. So we get from Equations (6) and (11):

$$
\begin{aligned}
& \nabla^{2} \boldsymbol{E}-g \mu \frac{\partial \boldsymbol{E}}{\partial t}-\varepsilon \mu \frac{\partial^{2} \boldsymbol{E}}{\partial t^{2}}=0, \\
& \nabla^{2} \boldsymbol{H}-g \mu \frac{\partial \boldsymbol{H}}{\partial t}-\varepsilon \mu \frac{\partial^{2} \boldsymbol{H}}{\partial t^{2}}=0 .
\end{aligned}
$$

In free space, i.e., in vacuum, what is also the inside of atoms is $g=0$, so the Equations (12) and (13) become:

$$
\begin{gathered}
\nabla^{2} \boldsymbol{E}-\varepsilon \mu \frac{\partial^{2} \boldsymbol{E}}{\partial t^{2}}=0, \\
\nabla^{2} \boldsymbol{H}-\varepsilon \mu \frac{\partial^{2} \boldsymbol{H}}{\partial t^{2}}=0,
\end{gathered}
$$

where, [9],

$$
\varepsilon \mu=\varepsilon_{\mathrm{r}} \varepsilon_{0} \mu_{\mathrm{r}} \mu_{0}=1 / u_{\mathrm{em}}^{2},
$$

and

$$
u_{\mathrm{em}}=\lambda v
$$

is phase velocity of electromagnetic wave, where $\lambda$ is the wavelength and $v$ is the frequency of the wave.

When processing the previous wave equations, there were no requirements that would exclude atoms. So you should take that Equations (14) and (15) are valid within the atom.

\subsection{Wave Equation on Lecher's Line}

Maxwell's equations and Kirchhoff's laws, [11,12], give the differential equation of voltage and current on Lecher's line (i.e., on parallel-wire transmission line, consisting of a pair of identic ideal conductive nonmagnetic parallel wires of radius $\rho$, separated by $\delta$, where the ratio $\delta / \rho=\chi$ ), Figure 1:

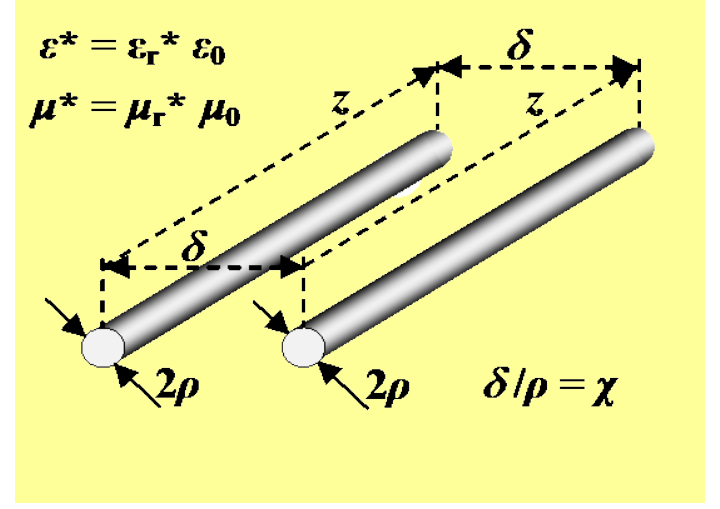

Figure 1. Lecher's line (section); twin-lead transmission line consisting of pair of ideal conductive nonmagnetic wires of diameter $2 \rho$, separated by $\delta$ situated in space with permittivity $\varepsilon^{*}=\varepsilon_{\mathrm{r}}^{*} \varepsilon_{0}$ and permeability $\mu^{*}=\mu_{\mathrm{r}}^{*} \mu_{0}$. 


$$
\begin{gathered}
\frac{\partial^{2} u}{\partial z^{2}}-L^{\prime} C^{\prime} \frac{\partial^{2} u}{\partial t^{2}}=0, \\
\frac{\partial^{2} i}{\partial z^{2}}-L^{\prime} C^{\prime} \frac{\partial^{2} i}{\partial t^{2}}=0,
\end{gathered}
$$

where $L^{\prime}$ is inductance of Lecher's line per unit length, and $C^{\prime}$ is its capacitance per unit length $[7,13]$, Figure 2:

$$
\begin{gathered}
L^{\prime}=\mu_{\mathrm{r}}^{*} \mu_{0} \frac{\ln \chi+1 / 4}{\pi}, \\
C^{\prime}=\varepsilon_{\mathrm{r}}^{*} \varepsilon_{0} \frac{\pi}{\ln \left(\chi / 2+\sqrt{(\chi / 2)^{2}-1}\right)},
\end{gathered}
$$

where $\varepsilon_{\mathrm{r}}{ }^{*}$ is relative permittivity and $\mu_{\mathrm{r}}{ }^{*}$ is relative permeability in the space of Lecher's line $\left(\varepsilon^{*}=\varepsilon_{\mathrm{r}}{ }^{*} \varepsilon_{0}, \mu^{*}=\right.$ $\left.\mu_{\mathrm{r}}^{*} \mu_{0}\right)$.

\section{Analogy between the Atom and Transmission Line}

For electromagnetic wave in an atom [Equations (14) and (15)], and for the wave of voltage and current on the transmission line (voltage/current wave) [Equations (18) and (19)], the same differential equations actually apply [14]. Physical phenomena, described using the same differential equations, behave the same. This basically means that a study of voltages and currents at the Lecher's line may conclude the behavior of electromagnetic waves in an atom. In this case, the voltage $u(z, t)$ on the line will represent the electric field $E_{\mathrm{x}}(z, t)$ of the electromagnetic wave, while the current line $i(z, t)$ to represent $H_{\mathrm{y}}(z, t)$ the magnetic field of the electromagnetic wave [14]. Take $H_{y}(z, t)$ and $i(z, t)$ :

$$
\begin{aligned}
& \frac{\partial^{2} H_{\mathrm{y}}(z, t)}{\partial z^{2}}-\varepsilon \mu \frac{\partial^{2} H_{\mathrm{y}}(z, t)}{\partial t^{2}}=0, \\
& \frac{\partial^{2} i(z, t)}{\partial z^{2}}-L^{\prime} C^{\prime} \frac{\partial^{2} i(z, t)}{\partial t^{2}}=0 .
\end{aligned}
$$

Now apply same relations to the electric field $E_{\mathrm{x}}(z, t)$ of the electromagnetic wave and voltage $u(z, t)$ the Lecher's line, i.e.,

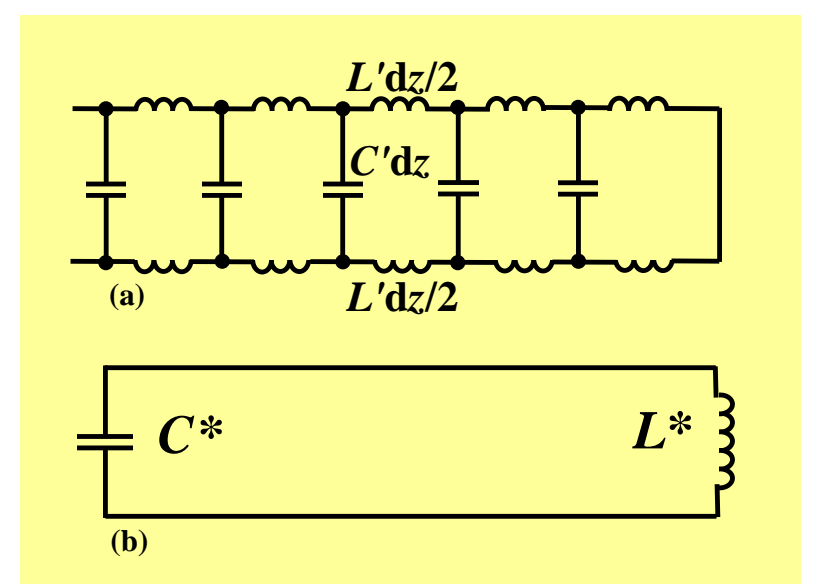

Figure 2. Lecher's line is presented by an infinite number of extremely small uniformly distributed capacitors, with capacitance $C^{\prime} \mathrm{dz}$, and inductors, with inductance $L^{\prime} \mathrm{dz}$, (a); all these capacitances are collected at the open end of the line, denoted by $C^{*}$, and inductances on its short-circuited end, denoted by $L^{*}$, resulting in a $L C$ circuit, (b). 


$$
\begin{gathered}
\frac{\partial^{2} E_{\mathrm{x}}(z, t)}{\partial z^{2}}-\varepsilon \mu \frac{\partial^{2} E_{\mathrm{x}}(z, t)}{\partial t^{2}}=0, \\
\frac{\partial^{2} u(z, t)}{\partial z^{2}}-L^{\prime} C^{\prime} \frac{\partial^{2} u(z, t)}{\partial t^{2}}=0 .
\end{gathered}
$$

Below we determine the parameters of Lecher's line, along which we will equate the behavior of electromagnetic waves in the atom and the voltage/current waves on Lecher's line by phase velocities, energies, frequencies and wavelengths.

\subsection{Phase Relations of Waves in the Atom and Voltage/Current Waves on the Lecher's Line}

Electromagnetic waves in the atom and the voltage/current wave on Lecher's line will behave equally in phase velocity if the phase velocity of the electromagnetic wave in an atom $u_{\mathrm{em}}$ is equal to the phase velocity of voltage/current wave on Lecher's line, $u_{\mathrm{em}}=u_{\mathrm{vc}}{ }^{*}$, i.e., in accordance with the Equations (22) and (23)

$$
\varepsilon \mu=L^{\prime} C^{\prime}
$$

From Equations (16), (17), (20) and (21) we get:

$$
\begin{gathered}
\varepsilon \mu=\varepsilon_{\mathrm{r}}^{*} \mu_{\mathrm{r}}^{*} \varepsilon_{0} \mu_{0} \frac{\ln \chi+\frac{1}{4}}{\ln \left(\frac{\chi}{2}+\sqrt{\frac{\chi^{2}}{4}-1}\right)}=\frac{\varepsilon_{\mathrm{r}}^{*} \mu_{\mathrm{r}}^{*} \varepsilon_{0} \mu_{0}}{[F(\chi)]^{2}}, \\
u_{\mathrm{em}}=\frac{1}{\sqrt{\varepsilon \mu}}=u_{\mathrm{vc}}^{*}=\frac{F(\chi)}{\sqrt{\varepsilon_{\mathrm{r}}^{*} \varepsilon_{0} \mu_{\mathrm{r}}^{*} \mu_{0}}}=\frac{c F(\chi)}{\sqrt{\varepsilon_{\mathrm{r}}^{*} \mu_{\mathrm{r}}^{*}}},
\end{gathered}
$$

where $c=\left(\varepsilon_{0} \mu_{0}\right)^{-2}$ is speed of light in vacuum and in accordance with Equations (25) and (26) is

$$
\begin{aligned}
& F(\chi)=\sqrt{\frac{\ln \left(\chi / 2+\sqrt{\chi^{2} / 4-1}\right)}{\ln \chi+1 / 4},} \\
& F(\chi)=u_{\mathrm{em}} \sqrt{\varepsilon_{\mathrm{r}}^{*} \mu_{\mathrm{r}}^{*} \varepsilon_{0} \mu_{0}}=\frac{u_{\mathrm{em}} \sqrt{\varepsilon_{\mathrm{r}}^{*} \mu_{\mathrm{r}}^{*}}}{c},
\end{aligned}
$$

provided that $\varepsilon_{\mathrm{r}}^{*}=\mu_{\mathrm{r}}^{*}$. Solving next two equations with two unknowns, i.e., $\mu^{*} / \varepsilon^{*}=\mu_{0} / \varepsilon_{0}$ and $F(\chi)=$ $\left(\varepsilon^{*} \mu^{*}\right)^{1 / 2} u_{\text {em }}$, we obtain:

$$
\begin{aligned}
& \varepsilon^{*}=\varepsilon_{\mathrm{r}}^{*} \varepsilon_{0}=\sqrt{\frac{\varepsilon_{0}}{\mu_{0}}} \frac{F(\chi)}{u_{\mathrm{em}}}, \\
& \mu^{*}=\mu_{\mathrm{r}}^{*} \mu_{0}=\sqrt{\frac{\mu_{0}}{\varepsilon_{0}}} \frac{F(\chi)}{u_{\mathrm{em}}}, \\
& \varepsilon_{\mathrm{r}}^{*}=\mu_{\mathrm{r}}^{*}=\frac{1}{\sqrt{\varepsilon_{0} \mu_{0}}} \frac{F(\chi)}{u_{\mathrm{em}}}=\frac{C}{u_{\mathrm{em}}} F(\chi) .
\end{aligned}
$$

\subsection{The Energy of the Electromagnetic Wave in the Atom and the Energy of the Voltage/Current Waves on Lecher's Line}

The total electromagnetic energy density, [6], is $(E \cdot D+B \cdot H) / 2$. Electromagnetic energy, i.e., the energy of an electromagnetic wave in an atom $E_{\mathrm{em}}$ is obtained by integrating this expression at a time throughout the entire space of the wave:

$$
E_{\mathrm{em}}=\frac{1}{2} \int(\boldsymbol{E} \cdot \boldsymbol{D}+\boldsymbol{B} \cdot \boldsymbol{H}) \mathrm{d} x \mathrm{~d} y \mathrm{~d} z .
$$


On the other hand electromagnetic energy in the atom is obtained also by using the energy balance in the atom. From the balance of forces in the atom follows [15]:

$$
\frac{m v^{2}}{r \sqrt{1-\beta^{2}}}=\frac{|q Q|}{4 \pi \varepsilon r^{2}} ; r=\frac{|q Q|}{4 \pi \varepsilon m c^{2}} \frac{\sqrt{1-\beta^{2}}}{\beta^{2}},
$$

where $r$ is the radius of the circular orbit of the electron, $q$ is the charge of the electron $(q=-e), e$ is elementary charge, $Q$ is the charge of the nucleus $(Q=Z e), Z$ is atomic number (which theoretically is not in integer domain), $m$ is the electron rest mass, $c=1 /\left(\mu_{0} \varepsilon_{0}\right)^{1 / 2}$ is the speed of light in vacuum, $\beta=v / c$, where $v$ is the velocity of the electron, $\varepsilon=\varepsilon_{\mathrm{r}} \varepsilon_{0}$, as stated above is permittivity, $\varepsilon_{\mathrm{r}}$ is relative permittivity, $\varepsilon_{0}$ is permittivity of free space, $\mu=\mu_{\mathrm{r}} \mu_{0}$, as stated above is permeability, $\mu_{\mathrm{r}}$ is relative permeability, $\mu_{0}$ is permeability of free space, the transverse mass of the electron is $m /\left(1-\beta^{2}\right)^{1 / 2}$, [16]. Increase of transverse mass of the electron is $\Delta m=m /\left(1-\beta^{2}\right)^{1 / 2}-m$. The kinetic energy of the electron, [15], is $K=\Delta m c^{2}$, Figure 3:

$$
K=\frac{m c^{2}}{\sqrt{1-\beta^{2}}}-m c^{2}
$$

Using Equation (31) and noting that an electron holds an opposite charge to the nucleus, the potential energy of electron, [12,15], is:

$$
U=\frac{q Q}{4 \pi \varepsilon r}=-\frac{m c^{2}}{\sqrt{1-\beta^{2}}} \beta^{2} .
$$

The total mechanical energy of an electron $W$, [15], is the sum of its kinetic and potential energies:

$$
W=K+U=-m c^{2}\left(1-\sqrt{1-\beta^{2}}\right),
$$

and according to the law of conservation of energy is equal to the negative emitted electromagnetic energy, $E_{\mathrm{em}}$,

$$
E_{\mathrm{em}}=-W=m c^{2}\left(1-\sqrt{1-\beta^{2}}\right)=e V,
$$

where $V$ is the potential difference through which the electron passes to get an equal energy as electromagnetic energy.

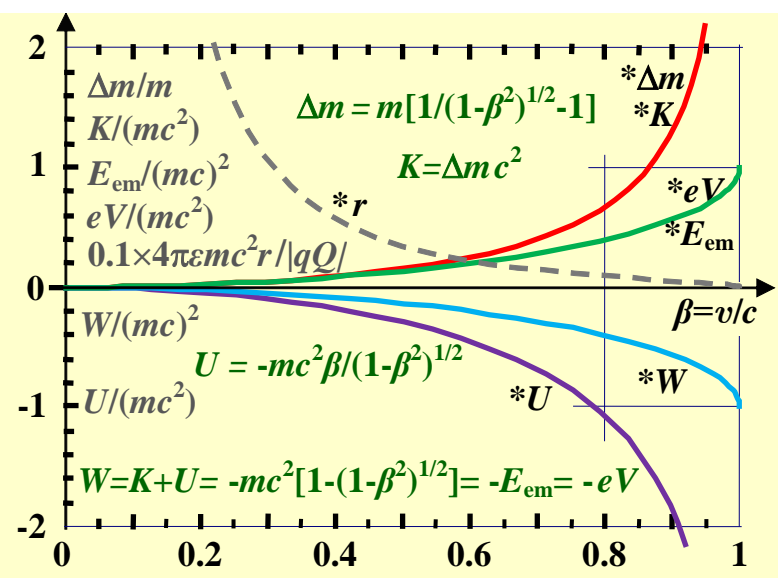

Figure 3. Energy relations in an atom (these relationships are valid for both classical and quantum physics; please note that the normalized quantities are marked with $*$ ). Radius of the circular orbit of the electron ${ }^{*} r=r /\left[10|q Q| /\left(4 \pi \varepsilon m c^{2}\right)\right]$, kinetic energy of the electron $* K=K /\left(m c^{2}\right)$, potential energy of the electron $* U=U /\left(m c^{2}\right)$, total mechanical energy of the electron $* W=* K+* U$, electromagnetic energy of the atom $* E_{\mathrm{em}}$ $=-* W=* e V$; all versus normalized velocity of the electron $\beta$ $=v / c$. 
Out of the Equation (35), $E_{\mathrm{em}}=m c^{2}\left[1-\left(1-\beta^{2}\right)^{1 / 2}\right]$, comes $\left(1-\beta^{2}\right)^{1 / 2}=1-E_{\mathrm{em}} / m c^{2}$ and $\beta^{2}=2 E_{\mathrm{em}}\left(1-E_{\mathrm{em}} / 2 m c^{2}\right) / m c^{2}$, so the radius $r$ in Equation (31) through arranging leads to:

$$
r=\frac{|q Q|}{8 \pi \varepsilon E_{\mathrm{em}}} \frac{1-E_{\mathrm{em}} / m c^{2}}{1-E_{\mathrm{em}} / 2 m c^{2}},
$$

or

$$
E_{\mathrm{em}}=\frac{1}{2} \frac{|q Q|}{4 \pi \varepsilon r} \frac{1-E_{\mathrm{em}} / m c^{2}}{1-E_{\mathrm{em}} / 2 m c^{2}}=\frac{1}{2}|U| \frac{1-E_{\mathrm{em}} / m c^{2}}{1-E_{\mathrm{em}} / 2 m c^{2}} .
$$

On the other hand Lecher's line can be shown as the inductive-capacitive network (so-called $L C$ network), which finally makes the oscillatory circuit ( $L C$ circuit), Figure 2, [11]. The natural frequency $v^{*}$ of $L C$ circuit is [15]:

$$
v^{*}=\frac{1}{2 \pi \sqrt{L^{*} C^{*}}},
$$

where $C^{*}$ is the sum of all small capacitances of the $L C$ network on the open end of the network, and $L^{*}$ is sum of all small inductances of the $L C$ network on the short-circuited of the network [11]. To realize the analogy between atoms and transmission lines according to the model presented in [14], electromagnetic energy in the atom $E_{\mathrm{em}}$ can be described as the electromagnetic energy of $L C$ circuit, i.e.,

$$
E_{\mathrm{em}}=\frac{1}{2} \frac{\Theta^{2}}{C^{*}},
$$

where $\Theta$ is maximal charge on the said capacitor $C^{*}$. Thus, using Equations (37) and (39) we obtain:

$$
\frac{1}{2} \frac{|q Q|}{4 \pi \varepsilon r} \frac{1-E_{\mathrm{em}} / m c^{2}}{1-E_{\mathrm{em}} / 2 m c^{2}}=\frac{1}{2} \frac{\Theta^{2}}{C^{*}} .
$$

The single Equation (40) has two unknowns, i.e., parameter $C^{*}$ and variable $\Theta$. By using Diophantine equations we get one of the many solutions [17]:

$$
\begin{gathered}
C^{*}=4 \pi \varepsilon r, \\
\Theta^{2}=|q Q| \frac{1-E_{\mathrm{em}} / m c^{2}}{1-E_{\mathrm{em}} / 2 m c^{2}} .
\end{gathered}
$$

\subsection{The Frequency of the Electromagnetic Wave in the Atom and the Frequency of Voltage/Current Waves on Lecher's Line}

Equation (40), which represents the electromagnetic energy in an atom $E_{\mathrm{em}}$, can be written like this:

$$
\frac{1}{2} \frac{\Theta^{2}}{C^{*}}=\frac{1}{2} \frac{\pi}{\pi} \frac{\Theta^{2}}{\sqrt{C^{*}} \sqrt{C^{*}}} \frac{\sqrt{L^{*}}}{\sqrt{L^{*}}}=\pi \sqrt{\frac{L^{*}}{C^{*}}} \Theta^{2} \frac{1}{2 \pi \sqrt{L^{*} C^{*}}}=\pi Z_{\mathrm{LC}}^{*} \Theta^{2} v^{*}=\pi Z_{\mathrm{LC}}^{*}|q Q| \frac{1-E_{\mathrm{em}} / m c^{2}}{1-E_{\mathrm{em}} / 2 m c^{2}} v^{*}=A v^{*},
$$

where

$$
A=\pi Z_{\mathrm{LC}}^{*}|q Q| \frac{1-E_{\mathrm{em}} / m c^{2}}{1-E_{\mathrm{em}} / 2 m c^{2}}
$$

is the action of the electromagnetic oscillator, and

$$
Z_{\mathrm{LC}}^{*}=\sqrt{\frac{L^{*}}{C^{*}}}=\sqrt{\frac{L^{\prime} \mathrm{d} z}{C^{\prime} \mathrm{d} z}}=\sqrt{\frac{L^{\prime}}{C^{\prime}}}=\sqrt{\frac{\mu^{*}}{\varepsilon^{*}}} \frac{\sqrt{\left[\ln \left(\chi / 2+\sqrt{\chi^{2} / 4-1}\right)\right](\ln \chi+1 / 4)}}{\pi}=\sqrt{\frac{\mu^{*}}{\varepsilon^{*}}} \frac{\sigma(\chi)}{\pi}
$$

is the characteristic impedance of Lecher's line, while 


$$
\sigma(\chi)=\sqrt{\left[\ln \left(\chi / 2+\sqrt{\chi^{2} / 4-1}\right)\right](\ln \chi+1 / 4)}
$$

is the structural coefficient of Lecher's line.

From Equations (40) and (43), in fact from $E_{\mathrm{em}}=\Theta^{2} /\left(2 C^{*}\right)=\pi Z^{*}{ }_{\mathrm{LC}} \Theta^{2} v^{*}$, furthermore from Equations (41) and (45), follows the natural frequency of voltage/current wave on Lecher's line $v^{*}$, which is analogous to the frequency $v$ of the electromagnetic wave in the atom

$$
v=v^{*}=\frac{1}{2 \pi Z_{\mathrm{LC}}^{*} C^{*}}=\frac{1}{8 \pi \varepsilon \sqrt{\mu^{*} / \varepsilon^{*}} \sigma(\chi) r} .
$$

Therefore in an atom with multiple electrons there are multiple natural frequencies. It should be noted that the same Equation as (47) came from simultaneously multiplying and dividing the right side of the Equation (38), i.e., $v^{*}=\left[2 \pi\left(L^{*} C^{*}\right)^{1 / 2}\right]^{-1}$, with $C^{* 1 / 2}$, and then using the expression $C^{*}=4 \pi \varepsilon r$ and Equation (45).

Equation (47) shows that the natural frequency $v^{*}$ does not depend on the amount of charge, but depends on the properties of the space in an atom $(\varepsilon)$ and in surrounding of Lecher's line $\left(\varepsilon^{*}, \mu^{*}\right)$, structural coefficient of Lecher's line $\sigma(\chi)$, (which for its part depends only on the parameters of Lecher's line $\delta$ and $\rho$ ), and the radius $r$ of the circular orbit of the electron. Indeed, electromagnetic oscillations require a charge, but amount of the charge does not affect the amount of the frequency.

If Equation (36) is inserted in Equation (47) we obtain natural frequency of electromagnetic wave in an atom:

$$
v=\frac{E_{\mathrm{em}}}{\sqrt{\mu^{*} / \varepsilon^{*}} \sigma(\chi)|q Q|} \frac{1-E_{\mathrm{em}} / 2 m c^{2}}{1-E_{\mathrm{em}} / m c^{2}} .
$$

\section{Subsequent Physical Quantities in the Atom}

\subsection{Structural Constant of the Atom}

In the Equation (48) charges $q$ and $Q$ appear in the form of the product $|q Q|$. In order to satisfy the condition that the frequency $v$ is independent of the charge, and to avoid direct or indirect involvement of the charge, the total product $\left(\mu^{*} / \varepsilon^{*}\right)^{1 / 2} \sigma(\chi)|q Q|=\left(\mu^{*} / \varepsilon^{*}\right)^{1 / 2} \sigma(\chi) Z e^{2}$ in Equation (48) must be a constant, i.e., only a product $\sigma(\chi) Z$ must be constant, because $\mu^{*} / \varepsilon^{*}=\mu_{0} / \varepsilon_{0}$, (it is assumed that $\varepsilon_{\mathrm{r}}^{*}=\mu_{\mathrm{r}}^{*}$ ) and $e$ are already considered as constants. This product, in the form

$$
s_{0}=\sqrt{\sigma(\chi) Z}=\text { const. }
$$

is called the structural constant of the atom.

\subsection{Action Constant of the Atom}

From Equations (44), (45) and (49) follows

$$
\begin{gathered}
A=\sqrt{\frac{\mu^{*}}{\varepsilon^{*}}} s_{0}^{2} e^{2} \frac{1-E_{\mathrm{em}} / m c^{2}}{1-E_{\mathrm{em}} / 2 m c^{2}}=A_{0} \frac{1-E_{\mathrm{em}} / m c^{2}}{1-E_{\mathrm{em}} / 2 m c^{2}}, \\
\frac{A}{A_{0}}=\frac{1-E_{\mathrm{em}} / m c^{2}}{1-E_{\mathrm{em}} / 2 m c^{2}},
\end{gathered}
$$

where

$$
A_{0}=\pi Z_{\mathrm{LC}}^{*}|q Q|=\sqrt{\frac{\mu_{\mathrm{r}}^{*} \mu_{0}}{\varepsilon_{\mathrm{r}}^{*} \varepsilon_{0}}} s_{0}^{2} e^{2}=\sqrt{\frac{\mu_{0}}{\varepsilon_{0}}} s_{0}^{2} e^{2},
$$

is action constant. Electromagnetic energy in accordance with the Equations (39), (40), (41) and (47), i.e.,

$$
E_{\mathrm{em}}=A v \text {, }
$$

using Equation (50), we can write as: 


$$
E_{\mathrm{em}}=A_{0} v \frac{1-E_{\mathrm{em}} / m c^{2}}{1-E_{\mathrm{em}} / 2 m c^{2}}
$$

By solving this equation we obtain:

$$
\begin{aligned}
& E_{\mathrm{em}}=A_{0} v+m c^{2}-\sqrt{\left(A_{0} v\right)^{2}+\left(m c^{2}\right)^{2}}, \\
& A=A_{0}+m c^{2} / v-\sqrt{A_{0}^{2}+\left(m c^{2} / v\right)^{2}} .
\end{aligned}
$$

Taking into account that $E_{\mathrm{em}}=e V$ it is as follows:

$$
e V / m c^{2}=A_{0} v / m c^{2}+1-\sqrt{\left(A_{0} v / m c^{2}\right)^{2}+1} .
$$

The extended Duane-Hunt's law we get from Equations (50) and (53) using $E_{\mathrm{em}}=e V$, [17], and using $\left(1-\beta^{2}\right)^{1 / 2}=1-E_{\mathrm{em}} / m c^{2}$ and $\beta^{2}=2 E_{\mathrm{em}}\left(1-E_{\mathrm{em}} / 2 m c^{2}\right) / m c^{2}$ we get also a portion $A_{0} v$ :

$$
\begin{gathered}
v=\frac{e V}{A_{0}} \frac{1-e V / 2 m c^{2}}{1-e V / m c^{2}} ; \\
v=\frac{e V}{A} ; \quad A_{0} v=\frac{1}{2} \frac{m}{\sqrt{1-\beta^{2}}} v^{2} .
\end{gathered}
$$

From Equations (37) and (53) follows:

$$
v=\frac{|U|}{2 A_{0}} .
$$

\subsection{Wavelength and Momentum of Electromagnetic Wave in the Atom}

The momentum of the electromagnetic wave in the atom is equal to the momentum of a photon, [14-16], $p_{\mathrm{em}}=$ $E_{\mathrm{em}} / u_{\mathrm{em}}=E_{\mathrm{em}} /(\lambda v)$, and according to the Equation (53) is, Figure 4,

$$
p_{\mathrm{em}}=\frac{E_{\mathrm{em}}}{\lambda v}=\frac{A_{0}}{\lambda} \frac{1-e V / m c^{2}}{1-e V / 2 m c^{2}}=\frac{A}{\lambda} .
$$

In accordance to the law of conservation of momentum, the momentum in Equation (58) is equal to the linear momentum of the electron, [14],

$$
\frac{m v}{\sqrt{1-\beta^{2}}}=\frac{m c \beta}{\sqrt{1-\beta^{2}}}=\frac{A}{\lambda} .
$$

By applying the expressions $\left(1-\beta^{2}\right)^{1 / 2}=1-E_{\mathrm{em}} / m c^{2}$ and $\beta^{2}=2 E_{\mathrm{em}}\left(1-E_{\mathrm{em}} / 2 m c^{2}\right) / m c^{2}$ to Equation (59) we obtain

$$
\lambda=\frac{A_{0}}{\sqrt{2 m e V}} \frac{\left(1-e V / m c^{2}\right)^{2}}{\sqrt{\left(1-e V / 2 m c^{2}\right)^{3}}},
$$

and using Equation (56) it becomes

$$
\lambda=\sqrt{\frac{A_{0}}{2 m v}} \frac{\sqrt{\left(1-e V / m c^{2}\right)^{3}}}{1-e V / 2 m c^{2}} .
$$

Phase velocity of the electromagnetic wave in an atom is obtained by multiplying two Equations, (56) and (60):

$$
u_{\mathrm{em}}=\lambda v=\sqrt{\frac{e V}{2 m}} \frac{1-e V / m c^{2}}{\sqrt{1-e V / 2 m c^{2}}} .
$$

From Equations (58) and (60) follows

$$
p_{\mathrm{em}}=\sqrt{2 m e V} \frac{\sqrt{1-e V / 2 m c^{2}}}{1-e V / m c^{2}} .
$$




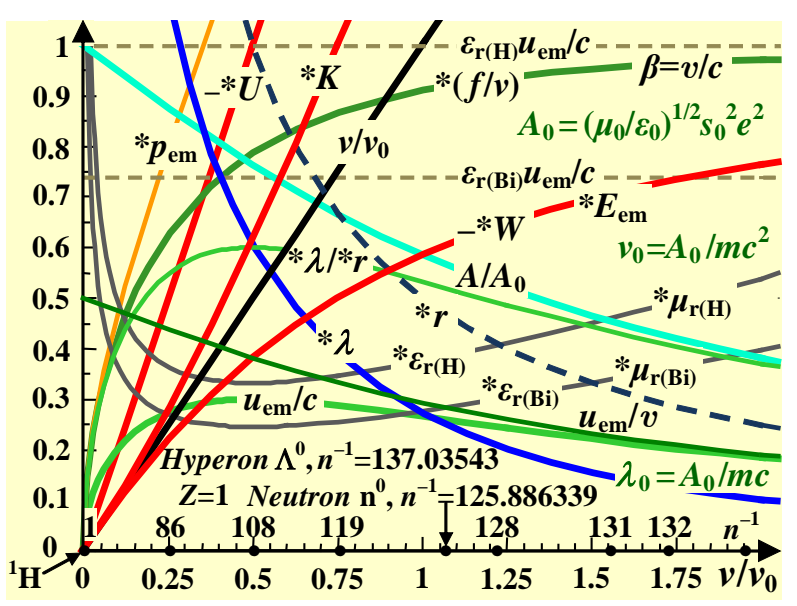

Figure 4. (Please note that the normalized quantities are marked on his left side with *). Radius of the circular orbit of the electron $* r=r /\left[|q Q| /\left(4 \pi \varepsilon m c^{2}\right)\right]$, normalized velocity of the electron $\beta=v / c$, kinetic energy of the electron $* K=K /\left(m c^{2}\right)$, potential energy of the electron $* U=U /\left(m c^{2}\right)$, total mechanical energy of the electron $* W=* K+* U$, electromagnetic energy of the atom $* E_{\mathrm{em}}=-* W$, momentum of electromagnetic wave in the atom ${ }^{*} p_{\mathrm{em}}=p_{\mathrm{em}} /(m c)$, wavelength of the electromagnetic wave in the atom $* \lambda=\lambda /\left[A_{0} /\left(m c^{2}\right)\right]$, normalized phase velocity of the electromagnetic wave in the atom $u_{\mathrm{em}} / c$ and $u_{\mathrm{em}} / v$, normalized action of the electromagnetic oscillator $A / A_{0}$, relative permittivity and relative permeability of the space in the hydrogen atom ${ }^{*} \varepsilon_{\mathrm{r}(\mathrm{H})}=*^{*} \mu_{\mathrm{r}(\mathrm{H})}=\varepsilon_{\mathrm{r}(\mathrm{H})} / 10=$ $\mu_{\mathrm{r}(\mathrm{H})} / 10$ and also relative permeability and relative permeability of the space within atom of bismuth $*_{\varepsilon_{\mathrm{r}(\mathrm{Bi})}}=*_{\mu_{\mathrm{r}(\mathrm{Bi})}}=$ $\varepsilon_{\mathrm{r}(\mathrm{Bi})} / 10=\mu_{\mathrm{r}(\mathrm{Bi})} / 10$, product of relative permittivity and normalized phase velocity $\varepsilon_{\mathrm{r}(\mathrm{H})} u_{\mathrm{em}} / c=\mu_{\mathrm{r}(\mathrm{H})} u_{\mathrm{em}} / c$ in the case of hydrogen, product of relative permittivity and normalized phase velocity $\varepsilon_{\mathrm{r}(\mathrm{Bi})} u_{\mathrm{em}} / c=\mu_{\mathrm{r}(\mathrm{Bi})} u_{\mathrm{em}} / c$ in the case of bismuth, the ratio of wavelength and radius $* \lambda / * r=(\lambda / r) /\left(4 \pi \varepsilon c A_{0} /|q Q|\right)=2 u_{\mathrm{em}} / c$, the ratio of the frequency $f$ of rotation of the electron orbiting atom and the frequency $v$ of the electromagnetic wave $*(f / v)=$ $(f / v) /\left(4 \varepsilon c A_{0} /|q Q|\right)$, all versus normalized frequency of the electromagnetic wave in the atom $v / v_{0}$, where $v_{0}=A_{0} / m c^{2} ; n^{+1}$ $=1,2,3, \ldots$ is ordinal number of stationary orbits in the atom, $n^{-1}=1,2,3, \ldots$, for hyperon $\Xi^{0} n^{-1}=137.03587$.

The ratio of the wavelength of the electromagnetic wave in the atom and the atom radius are obtained from Equations (36) and (60) using $E_{\mathrm{em}}=e V$ and Equation (62), Figure 4:

$$
\frac{\lambda}{r}=\frac{8 \pi \varepsilon A_{0}}{|q Q|} \sqrt{\frac{e V}{2 m}} \frac{1-e V / m c^{2}}{1-e V / 2 m c^{2}}=\frac{8 \pi \varepsilon A_{0}}{|q Q|} u_{\mathrm{em}}
$$

This expression, with Equation (33), $|U|=|q Q| /(4 \pi \varepsilon r)$, leads to:

$$
\lambda=2 A_{0} \frac{u_{\mathrm{em}}}{|U|} \text {. }
$$

Equation (65) is confirmed by Equations (17) and (57).

\subsection{Synchronized (Stationary) States of Atoms}

A minimum of two separate oscillating processes are performed simultaneously within an atom, i.e., the circular motion of electrons around the nucleus and oscillation of electromagnetic wave energy [3]. The time period of 
one circular tour of electrons around the nucleus is $T_{\mathrm{e}}=2 r \pi / v=1 / f$, where $f$ is the frequency of circulation of electrons around the nucleus. The duration of the period of the electromagnetic wave is $T_{\mathrm{em}}=1 / v$. Hence, $v / f=$ $2 \pi v r / v$. Using Equation (64), as well as $v / c=\beta$ and $\lambda v=u_{\mathrm{em}}$ follows, Figure 4:

$$
\begin{aligned}
& \frac{T_{\mathrm{e}}}{T_{\mathrm{em}}}=\frac{v}{f}=\frac{|q Q|}{4 \varepsilon A_{0} v}, \\
& \frac{f}{v}=\frac{4 \varepsilon A_{0} c}{|q Q|} \beta .
\end{aligned}
$$

Long term existence of the rotation of electrons and long term existence of the electromagnetic wave in the atom (stationary state) is only possible if there is synchronism between them (synchronously stationary state) $[3,4]$. Namely, to be coherent with the active power of the electromagnetic wave in an atom, the electron needs to oscillate (i.e., rotate) with dual frequency of the wave, because the active power of wave oscillates with dual frequency $2 \omega=2(2 \pi v)$, (this will be further discussed in Sub-Heading 4.5). This means that in the synchronously stationary state of the atom, the time period of electron rotation $T_{\mathrm{e}}$ is a half period of $T_{\mathrm{em}}$ (or, for reasons of synchronism, is $n^{ \pm 1}$-multiple of a half period of $T_{\mathrm{em}}$ ), i.e., $T_{\mathrm{e}}=n^{ \pm 1} T_{\mathrm{em}} / 2$, where $n^{+1}=1,2,3, \ldots$ is ordinal number of stationary orbits in the atom, when an electron moves away from the nucleus (or $n^{-1}=1,2,3, \ldots$, when the electron approaches the nucleus). Equation (66) gives the speed of electron in a synchronously stationary state [4] (compare with [15]):

$$
v_{\mathrm{n}}=\frac{1}{n^{ \pm 1}} \frac{|q Q|}{2 \varepsilon A_{0}} .
$$

The Equations (31) and (67) give the radius of the electron orbits in the synchronously stationary states:

$$
r_{\mathrm{n}}=\left(n^{ \pm 1}\right)^{2} \frac{\varepsilon A_{0}^{2} \sqrt{1-\left(v_{\mathrm{n}} / c\right)^{2}}}{\pi m|q Q|} .
$$

From Equations (66), (67) and (68) follows [4]:

$$
\begin{aligned}
& f_{\mathrm{n}}=\frac{1}{\left(n^{ \pm 1}\right)^{3}} \frac{|q Q|^{2} m}{4 \varepsilon^{2} A_{0}^{3} \sqrt{1-\left(v_{\mathrm{n}} / c\right)^{2}}}, \\
& v_{\mathrm{n}}=\frac{1}{\left(n^{ \pm 1}\right)^{2}} \frac{|q Q|^{2} m}{8 \varepsilon^{2} A_{0}^{3} \sqrt{1-\left(v_{\mathrm{n}} / c\right)^{2}}}
\end{aligned}
$$

and, [3],

$$
f_{\mathrm{n}}=2\left(\frac{v_{\mathrm{n}}}{n^{ \pm 1}}\right)
$$

The total mechanical energy of an electron $W_{\mathrm{n}}=-E_{\text {em(n) }}$ follows from Equations (54) and (70):

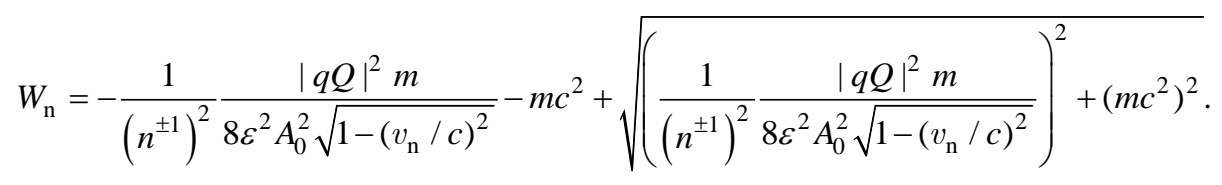

For energies much smaller than $m c^{2}$ :

$$
W_{\mathrm{n}} \approx-\frac{1}{\left(n^{ \pm 1}\right)^{2}} \frac{|q Q|^{2} m}{8 \varepsilon^{2} A_{0}^{2} \sqrt{1-\left(v_{\mathrm{n}} / c\right)^{2}}} .
$$

If assume the maximum speed of electron is equal to the speed of light in a given medium, i.e., according to Equation (28) $v_{\max }=u_{\mathrm{em}}=F(\chi) /\left(\mu^{*} \varepsilon^{*}\right)^{1 / 2}$ (to increase the speed of electron should be $n^{ \pm 1}=1 / n_{\max }$ ) from Equations (51) and (67) we get:

$$
n_{\max }=\frac{2 s_{0}^{2}}{Z} F(\chi)
$$


From Equation (74) follows the greatest possible atomic number $Z_{\max }$ when $n_{\max }$ is minimal and $F(\chi)$ is maximal, actually when $n_{\max }=1$ and $F(\chi)=1$, i.e.,

$$
Z_{\max }=\frac{2 s_{0}^{2}}{n_{\max }} F(\chi)=2 s_{0}^{2} .
$$

\subsection{Wave Equations of the Electromagnetic Wave in the Atom}

Wave equations of electromagnetic wave in an atom are expressed by Equations (14) and (15). If we insert phase velocity $u_{\mathrm{em}}$, expressed from Equation (62), i.e.,

$$
u_{\mathrm{em}}^{2}=\frac{e V}{2 m} \frac{\left(1-e V / m c^{2}\right)^{2}}{1-e V / 2 m c^{2}},
$$

in Equations (14) and (15), we obtain

$$
\begin{aligned}
& \nabla^{2} \boldsymbol{E}-\frac{2 m}{e V} \frac{1-e V / 2 m c^{2}}{\left(1-e V / m c^{2}\right)^{2}} \frac{\partial^{2} \boldsymbol{E}}{\partial t^{2}}=0, \\
& \nabla^{2} \boldsymbol{H}-\frac{2 m}{e V} \frac{1-e V / 2 m c^{2}}{\left(1-e V / m c^{2}\right)^{2}} \frac{\partial^{2} \boldsymbol{H}}{\partial t^{2}}=0 .
\end{aligned}
$$

Wave Equations (14), (15) or (77) have a lot of solutions. We will apply the solutions that correspond to the atom and the transmission line, i.e., to the $L C$ network. These solutions are standing waves $[6,8]$ :

$$
\begin{aligned}
& E_{\mathrm{x}}(z, t)=E_{0} \sin \left(\frac{2 \pi z}{\lambda}\right) \cos (\omega t), \\
& H_{\mathrm{y}}(z, t)=-\frac{E_{0}}{\sqrt{\mu / \varepsilon}} \cos \left(\frac{2 \pi z}{\lambda}\right) \sin (\omega t),
\end{aligned}
$$

where $E_{0}$ is the maximum value, i.e., the amplitude of electric field strength $E, E_{\mathrm{x}}(z, t)$ is the $x$-component of the electric field strength dependent on the $z$-axis and the time $t$, and $H_{\mathrm{y}}(z, t)$ is the $y$-component of the magnetic field strength $H$ dependent on the $z$-axis and the time $t, \omega=2 \pi v$. All mathematical operations we perform for the $y$-component of the magnetic field $H_{\mathrm{y}}(z, t)$ can be performed for the $x$-component of the electric field $E_{\mathrm{x}}(z, t)$ in the same way.

In the standing waves (78) the energy oscillates between the electric and magnetic form. The electrical energy is at a maximum when the magnetic energy is zero, and vice versa. Furthermore, the standing wave transfers no energy through the space because the average active power of the wave is equal to zero. The current value of the active power oscillates in both directions, + and - of $z$ axis, with dual frequency $2 \omega$ from point to point of $z$ axis [8]. As already mentioned, this is why (for the maintenance of stationary state of the atom) the electron has to rotate twice as fast compared to the lower harmonics $\left(n^{+1}\right)$, or twice as fast compared to the upper harmonics $\left(n^{-1}\right)$, i.e., $f=2\left(v / n^{ \pm 1}\right)$ in accordance with Equation (71).

If we use the second derivative with respect to $z$ of the $y$-component $H_{y}(z, t)$ of the magnetic field strength in Equation (78), we get: $\partial^{2} H_{y}(z, t) / \partial z^{2}+(2 \pi / \lambda)^{2} H_{y}(z, t)=0$. After inclusion of the wavelength $\lambda$ from Equation (60) we obtain:

$$
\frac{\partial^{2} H_{\mathrm{y}}(z, t)}{\partial \mathrm{z}^{2}}+\frac{8 \pi^{2} m e V}{A_{0}^{2}} \frac{\left(1-e V / 2 m c^{2}\right)^{3}}{\left(1-e V / m c^{2}\right)^{4}} H_{\mathrm{y}}(z, t)=0 .
$$

If $e V / m c^{2}<<1$, then $e V^{\prime \prime} K=W-U$, and Equation (79) becomes

$$
\frac{\partial^{2} H_{\mathrm{y}}(z, t)}{\partial z^{2}}+\frac{8 \pi^{2} m}{A_{0}^{2}}(W-U) H_{\mathrm{y}}(z, t)=0 .
$$

The second derivative of $H_{\mathrm{y}}(z, t)$ with respect to $t$ gives: 
$\partial^{2} H_{\mathrm{y}}(z, t) / \partial t^{2}+\omega^{2} H_{\mathrm{y}}(z, t)=0$. After inclusion of the angular frequency $\omega=2 \pi v$ from Equation (56) we obtain:

$$
\frac{\partial^{2} H_{\mathrm{y}}(z, t)}{\partial t^{2}}+\left(\frac{2 \pi}{A_{0}} e V \frac{1-e V / 2 m c^{2}}{1-e V / m c^{2}}\right)^{2} H_{\mathrm{y}}(z, t)=0 .
$$

If $e V / m c^{2}<<1$, then $e V^{\prime \prime} K=W-U$, and Equation (81) becomes

$$
\frac{\partial^{2} H_{\mathrm{y}}(z, t)}{\partial t^{2}}+\frac{4 \pi^{2}}{A_{0}^{2}}(W-U)^{2} H_{\mathrm{y}}(z, t)=0 .
$$

\section{Calculation of the Structural Constant $s_{0}$}

Only the structural constant $s_{0}$ of the atom is unknown in previous expressions. This constant can be determined in several ways, e.g., by measuring two quantities, the voltage $V$ and frequency $v$ and calculating the action constant $A_{0}$ by Duane-Hunt's law, i.e., using Equations (15) and (19), [17]. However, here we will use a more direct theoretical calculation, with only one empirical item necessary [18].

Namely, the increase of the nuclear charge in the atom increases atomic number $Z$. In accordance with Equation (49), the value of structural coefficient $\sigma(\chi)=s_{0}{ }^{2} / Z$ is assigned to each atom. So, greater atomic number means a lower structural coefficient $\sigma(\chi)$.

On the other hand, there is a critical nuclear charge which ensures stability of the atom $[9,19]$. In other words, reducing $\sigma(\chi)$ grows instability of the atom. In general, the higher atomic number means less stability (i.e., smaller half-life, or $\left.t_{1 / 2}\right)$ of the atom, starting from bismuth ${ }^{83} \mathrm{Bi}\left(Z=83, t_{1 / 2}=6 \times 10^{26} \mathrm{~s}\right.$, [16]) to ununoctium ${ }^{118}$ Uuo $\left(Z=118, t_{1 / 2}=5 \mathrm{~ms}\right)$, [exceptions are atoms of technetium $\left({ }^{43} \mathrm{Tc}, Z=43, t_{1 / 2}=1.3 \times 10^{14} \mathrm{~s}\right)$ and promethium $\left.\left({ }^{63} \mathrm{Pm}, Z=63, t_{1 / 2}=5.6 \times 10^{8} \mathrm{~s}\right)\right]$.

For the calculation of structural constant $s_{0}$ it is enough to find only one associated pair of $\sigma(\chi)$ and $Z$. The curve $\sigma(\chi)$ has no extremes, Figure 5. Thus it is not easy to find a mentioned pair of $\sigma(\chi)$ and $Z$. In that sense, a better situation is with the phase velocity $u_{\mathrm{em}}$, specifically with the normalized phase velocity $u_{\mathrm{em}}\left(\varepsilon^{*} \mu^{*}\right)^{1 / 2}=F(\chi)$ of electromagnetic wave in the atom, Figure 5, [9]. Neither of these two curves have extremes, but there is a sharp knee on $F(\chi)$ which can be used to determine the structural constant $s_{0}$.

Although there is no theory about the connection between the phase velocity of electromagnetic waves in the atom and the stability of the atoms, it is still possible to use this mathematical benefit of sharp knee for those

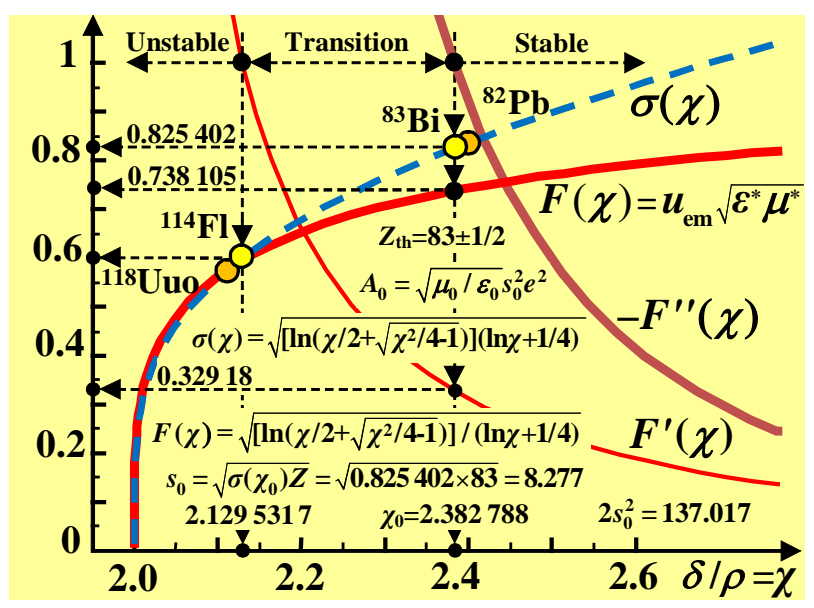

Figure 5. Structural coefficient of Lecher's line $\sigma(\chi)$, normalized phase velocity of the electromagnetic wave in the atom $F(\chi)=u_{\mathrm{em}}\left(\varepsilon^{*} \mu^{*}\right)^{1 / 2}$, the first derivative of the normalized phase velocity $F^{\prime}(\chi)$, inverted second derivative of the normalized phase velocity $F^{\prime \prime}(\chi)$, all versus ratio $\delta / \rho=\chi$ of the transmission Lecher's line, consisting of a pair of ideal conducting nonmagnetic parallel wires of radius $\rho$ separated by $\delta$, which represents a model of an atom. 
atoms, in which there is the lower phase velocity of electromagnetic waves that exhibit greater instability. Use of this result will be discussed just a little bit later.

The nuclear binding energy per nucleon slightly decreases with increasing atomic number (starting from the first radioactive element bismuth, ${ }^{83} \mathrm{Bi}, 7.848 \mathrm{MeV}$, to the ununoctium, ${ }^{118} \mathrm{Uuo}, 7.074 \mathrm{MeV}$, about $0.31 \%$ decrease for each of the 35 atoms in that area [20]). Physically this means that the boundary between stable and unstable areas is not emphasized. Mathematically it allows that between the two areas there exists so-called transition area, Figure 5. This is, at the moment, the most accurate way to determine the boundary between stable atoms and the others. Indeed, the first unstable atom must be located on that border. This is a key fact to determine the structural constant $s_{0}$ of the atom.

Before calculating, we observe the first derivative $F$ ' $(\chi)$ of the curves of normalized phase velocity $F(\chi)$ of electromagnetic waves in an atom (Figure 5). When this derivative is greater than 1, it means that the phase velocity rapidly declines, that is a zone of unstable atoms $(2<\chi<2.1295317)$. It should be noted that the situation $\chi \leq 2$ is theoretically impossible because then there is no Lecher's line.

When the second derivative $F^{\prime \prime}(\chi)$ of the normalized phase velocity $F(\chi)$ is greater than 1 , it means that the phase velocity starts to rapidly decline (Figure 5), this is a transition zone (2.129 $5317<\chi<2.382788)$.

The border crossing from the transition zone to the stable zone (i.e., $\chi_{0}=2.382788$ ), in accordance with the experiments, [20], is closest to the bismuth atom. Bismuth atom $\left({ }^{83} \mathrm{Bi}\right)$ is the first unstable atom, in the entire chain of stable atoms, which ends with lead $\left({ }^{82} \mathrm{~Pb}\right)$. The corresponding value of the structural coefficient in that place is $\sigma\left(\chi_{0}\right)=0.825402$, Figure 5. Bismuth is a chemical element with atomic number $Z=83$, with half-life more than a billion times the estimated age of the universe. Even though charges in reality take discrete values $\left(e, 2 e, 3 e, Z e\right.$ ), theoretical value of $Z$ in Equation (49) can be within the range $Z_{\mathrm{th}}=83 \pm 1 / 2$. Thus, according to Equation (49) we get the structural constant of the atom $[0.825402 \times(83 \pm 1 / 2)]^{1 / 2}$, i.e., $8.252<s_{0}<8.302$, with a mean value 8.277 and with sample standard deviation \pm 0.035355 or as a percentage $s_{0}=8.277 \pm 0.43 \%$. Comparing with the fine structure constant we get $s_{0}=8.27756$, which is consistent with the calculation performed here (the relative difference is less than $0.0068 \%$ ).

\section{System of the Elements}

After calculating the structural constants $s_{0}$ the structural coefficient $\sigma$ of each element can be determined. For this purpose we use Equations (46) and (49), Figure 6, i.e.,

$$
\sigma(\chi)=\sqrt{\left[\ln \left(\frac{\chi}{2}+\sqrt{\frac{\chi^{2}}{4}-1}\right)\right]\left(\ln \chi+\frac{1}{4}\right)}=\frac{s_{0}^{2}}{Z} .
$$

It can be seen that there is a maximal atomic number, 137, just as it is defined in Equation (75). Recently discovered element $(\mathrm{Z}=118)$ is ununoctium [Joint Institute for Nuclear Research (JINR) by Yuri Oganessian and his group in Dubna, Russia, 2002].

A century well-known behavior of hydrogen atoms describing Equations (70) and (72) if we put $n^{ \pm 1}=n^{+1}$ (in the case of $n^{ \pm 1}=1$ we get Lyman series, in case of $n^{ \pm 1}=2$ Balmer series, $n^{ \pm 1}=3$ Paschen series, $n^{ \pm 1}=4$ Brackett series, $n^{ \pm 1}=5$ Pfund series).

Interestingly, the same Equations (70) and (72) describe behavior of neutron, hyperon $\Lambda^{0}$ and hyperon $\Xi^{0}$ if we put $n^{ \pm 1}=n^{-1}$ (i.e., $n^{ \pm 1}=1 / 125.886339$ for neutron $n^{0}, n^{ \pm 1}=1 / 137.03543$ for hyperon $\Lambda^{0}, n^{ \pm 1}=1 / 137.03587$ for hyperon $\Xi^{0}$ ). This is the reason that all three of these particles are in the same place as hydrogen (Figure 6), [21].

To the similar results regarding the elementary particles otherwise on original way came Giuseppe Bellotti, $[22,23]$. He was also given new periodic classification of the elements on the basis of standing waves in the atom, using wave equation of potentials of the electron and positron, similar to the Equation (77) of electric and magnetic fields in this paper.

\section{Conclusions}

Maxwell's equations, together with the Lorentz equations, proved to suffice for the construction of a model of the atom. This model is made by analogy with the transmission line (Lecher's line). Using this model laws 


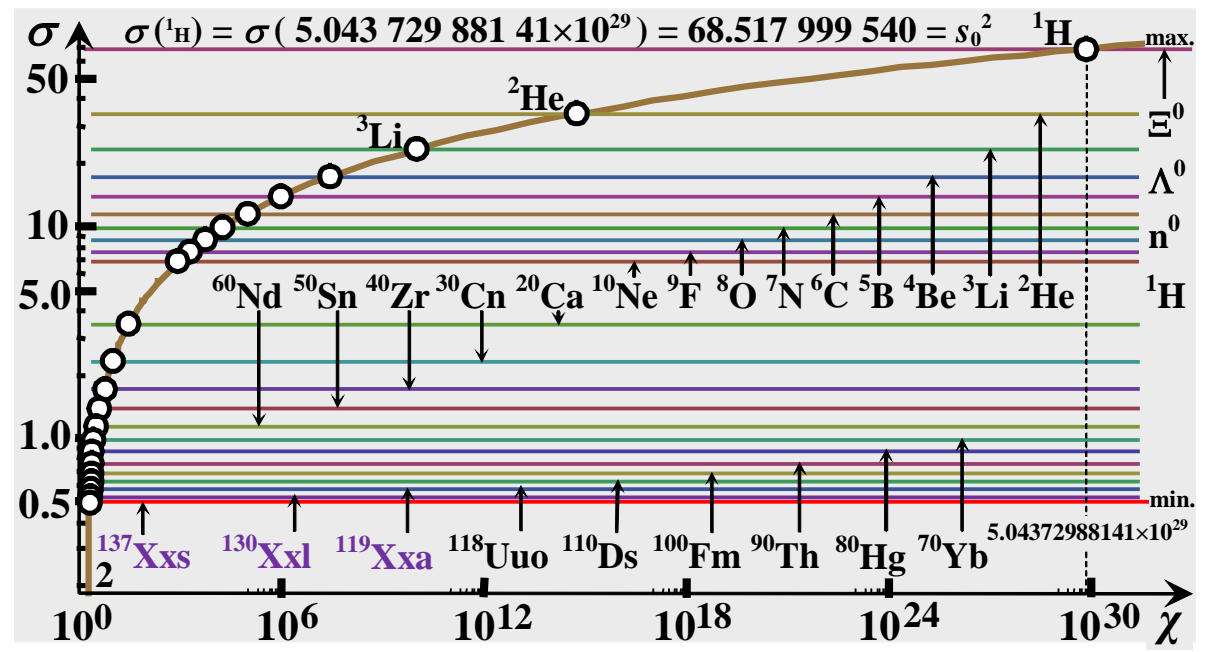

Figure 6. System of the elements, i.e., structural coefficient of Lecher's line $\sigma$ vs. parameter $\chi$ of Lecher's line, specifying all known elements, as well as 19 till now undiscovered elements, starting with atomic number 119, up to and including atomic number 137 (log-log scale). Recently discovered element, in the second year of two thousand, is ununoctium, $Z=118$, Dubna, Russia (Results from the first ${ }^{249} \mathrm{Cf}+{ }^{48} \mathrm{Ca}$ experiment). Each element has more stationary states which are determined with different amount of $n^{ \pm 1}$. So with $n^{ \pm 1}=1$ we obtain Lyman series of radiation of the hydrogen atom, with $n^{ \pm 1}=2$ Balmer series, with $n^{ \pm 1}=3$ Paschen series, with $n^{ \pm 1}=4$ Brackett series, with $n^{ \pm 1}=5$ Pfund series. If $n^{ \pm 1}=1 /(125.886339)$ hydrogen atom takes on the properties of neutron $n^{0}$, if $n^{ \pm 1}=1 /(137.03543)$ hydrogen atom takes on the properties of hyperon $\Lambda^{0}$, and if $n^{ \pm 1}=1 /(137.03587)$ hydrogen atom takes on the properties of hyperon $\Xi^{0}$. This is why $n^{0}, \Lambda^{0}$ and $\Xi^{0}$ are in the same place where there is hydrogen ${ }^{1} \mathrm{H}$. A minimal structural coefficient $\sigma_{\min }$ is obtained when the velocity of the electron is equal to the phase velocity of the electromagnetic wave in the atom, i.e., according to Equations (75) and (83) $\sigma_{\min }=s_{0}{ }^{2} / Z_{\max }=s_{0}{ }^{2} /\left(2 s_{0}{ }^{2}\right)=1 / 2$. The maximal amount of $\sigma$ arises, according to Equation (83), when the atomic number is the minimal $(Z=1)$, i.e. $\sigma_{\max }=s_{0}{ }^{2} / Z=s_{0}{ }^{2}=68.517999$ 540. All other elements are within this area.

which apply in quantum mechanics are derived, and are performed and Schrödinger's equation, with the clear meaning of the wave function. The wave function represents the electric or magnetic field strength of the electromagnetic wave in the atom.

Using the synchronization of two phenomena within the atom, the electromagnetic wave and the circular motion of electrons, stationary states of atoms are derived. It has been shown that two directions relative to the base state $(n=1)$ are possible. One is shift of the electron out of the center to the outside $\left(n^{ \pm 1}=n^{+1} ; n=1,2,3 \ldots\right)$, and the other is a shift in direction to the center of the atom $\left(n^{ \pm 1}=n^{-1} ; n=1,2,3 \ldots\right)$. The first is a classic, well-known for a hundred years. The latter is a novelty, and it makes possible the formation of neutrons and hyperons using protons and electrons, as is the case by hydrogen.

Structural constant of the atoms was determined with the aid of Maxwell's and Lorentz's equations. The amount of structural constant 8.27756 is determined by the rapid decline of the phase velocity of the electromagnetic wave in the atom. It happens to the bismuth atom.

Finally, it was found that the atomic number cannot exceed 137, meaning that it is still theoretically possible to detect another 19 so far undiscovered elements.

\section{Acknowledgements}

Wolfram Research, Inc. Mathematica software is used by courtesy of Systemcom, Ltd., Zagreb, Croatia, www.systemcom.hr. The author thanks Ms. Erica Vesic for editing this article in English, Mr. Damir Vuk and Mr. Branko Balon for the useful discussions, Prvomajska TZR, Ltd., Zagreb, Croatia, www.prvomajska-tzr.hr and Drives-Control, Ltd., Zagreb, Croatia, www.drivesc.com, for the financial support. 


\section{References}

[1] Wheeler, J.A. and Feynman, R.P. (1945) Interaction with the Absorber as the Mechanism of Radiation. Reviews of Modern Physics, 17, 157-181. http://dx.doi.org/10.1103/RevModPhys.17.157

[2] Wheeler, J.A. and Feynman, R.P. (1949) Classical Electrodynamics in Terms of Direct Interparticle Action. Reviews of Modern Physics, 21, 425-433.

[3] Perkovac, M. (2002) Quantization in Classical Electrodynamics. Physics Essays, 15, 41-60. http://dx.doi.org/10.4006/1.3025509

[4] Perkovac, M. (2003) Absorption and Emission of Radiation by an Atomic Oscillator. Physics Essays, 16, 162-173. http://dx.doi.org/10.4006/1.3025572

[5] Frankl, D.R. (1986) Electromagnetic Theory. Prentice-Hall, Inc., Englewood Cliffs, New Jersey.

[6] Jackson, J.D. (1999) Classical Electrodynamics. 3rd Edition, John Wiley \& Sons Inc., New York.

[7] Bosanac, T. (1973) Teoretska elektrotehnika 1. Tehnicka knjiga, Zagreb.

[8] Haznadar, Z. and Stih, Z. (1997) Elektromagnetizam. Skolska knjiga, Zagreb.

[9] Hänsel, H. and Neumann, W. (1995) Physik. Spektrum Akademischer Verlag, Heidelberg.

[10] Perkovac, M. (2012) Maxwell Equations for Nanotechnology. Proceedings of the 35th International Convention of the IEEE MIPRO, Opatija, 21-25 May 2012, 429-436. http://ieeexplore.ieee.org/xpl/freeabs_all.jsp?arnumber=6240683

[11] Rüdenberg, R. (1923) Elektrische Schaltvorgänge. Verlag von Julius Springer, Berlin.

[12] Czichos and Association Hütte (1989) Die Grundlagen der Ingenieurwissenschaften. Springer-Verlag, Berlin.

[13] Surutka, J. (1971) Elektromagnetika. Gradjevinska knjiga, Beograd.

[14] Perkovac, M. (2013) Model of an Atom by Analogy with the Transmission Line. Journal of Modern Physics, 4, 899903. http://dx.doi.org/10.4236/jmp.2013.47121

[15] Giancolli, D.C. (1988) Physics for Scientists and Engineers. Prentice Hall, Englewood Cliffs.

[16] Page, L. and Adams, N.I. (1940) Electrodynamics. D. Van Nostrand Company, Inc., New York.

[17] Perkovac, M. (2010) Statistical Test of Duane-Hunt’s Law and Its Comparison with an Alternative Law. http://arxiv.org/abs/1010.6083

[18] Perkovac, M. (2014) Determination of the Structural Constant of the Atom. Journal of Applied Mathematics and Physics, 2, 11-21. http://dx.doi.org/10.4236/jamp.2014.23002

[19] Benguria, R.D., Loss, M. and Siedentop, H. (2007) Stability of Atoms and Molecules in an Ultrarelativistic ThomasFermi-Weizsäcker Model, 1-11. http://dx.doi.org/10.1063/1.2832620

[20] http://periodictable.com/Isotopes/001.1/index.html

[21] http://www.physics.upenn.edu/ pgl/e27/E27.pdf

[22] Bellotti, G. (2012) The Hydrogen Atomic Model Based on the Electromagnetic Standing Waves and the Periodic Classification of the Elements. Applied Physics Research, 4, 141-151. http://dx.doi.org/10.5539/apr.v4n3p141

[23] Bellotti, G. (2012) The Ideas Behind the Electromagnetic Atomic Theory. Advances in Natural Science, 5, 7-11. http://cscanada.net/index.php/ans/article/view/j.ans.1715787020120504.2014/3315 